EPJ manuscript No.

(will be inserted by the editor)

\title{
Energy-weighted Sum Rules, y-scaling and Duality
}

\author{
J. W. Van Orden ${ }^{12}$ and Sabine Jeschonnek ${ }^{3}$ \\ 1 Department of Physics, Old Dominion University, Norfolk, VA 23529 USA \\ 2 Jefferson Lab, 12000 Jefferson Ave., Newport News, VA 23606, USA \\ 3 The Ohio State University, Physics Department, Lima, OH 45804, USA
}

September 16, 2002

\begin{abstract}
The phenomena of scaling and Bloom-Gilman duality are examined in the context of simple nonrelativistic and relativistic quantum mechanical models. These models are shown to scale and to show the qualitative features of Bloom-Gilman duality. This suggests that these phenomena do not necessarily require the properties of $\mathrm{QCD}$.
\end{abstract}

PACS. 12.40.Nn Regge theory, duality, absorptive/optical models - 12.39.Ki Relativistic quark model 13.60.Hb Total and inclusive cross sections

\section{Introduction}

The phenomena of scaling and Bloom-Gilman duality[1] are displayed by the data from Jefferson Lab[2] displayed in Fig. 1. Here the structure function $F_{2}^{p}$ is displayed for a variety of four-momentum transfers $Q^{2}$ as a function of the Nachtmann scaling variable $\xi[3,4]$. Scaling requires that the structure functions become independent of $Q^{2}$ (up to logarithmic corrections) for sufficiently high $Q^{2}$. The fit of teh NMC collaboration for $Q^{2}=5 \mathrm{GeV}^{2}$ is shown as the solid curve in this figure. The scaling region is usually associated with the disappearance of resonance

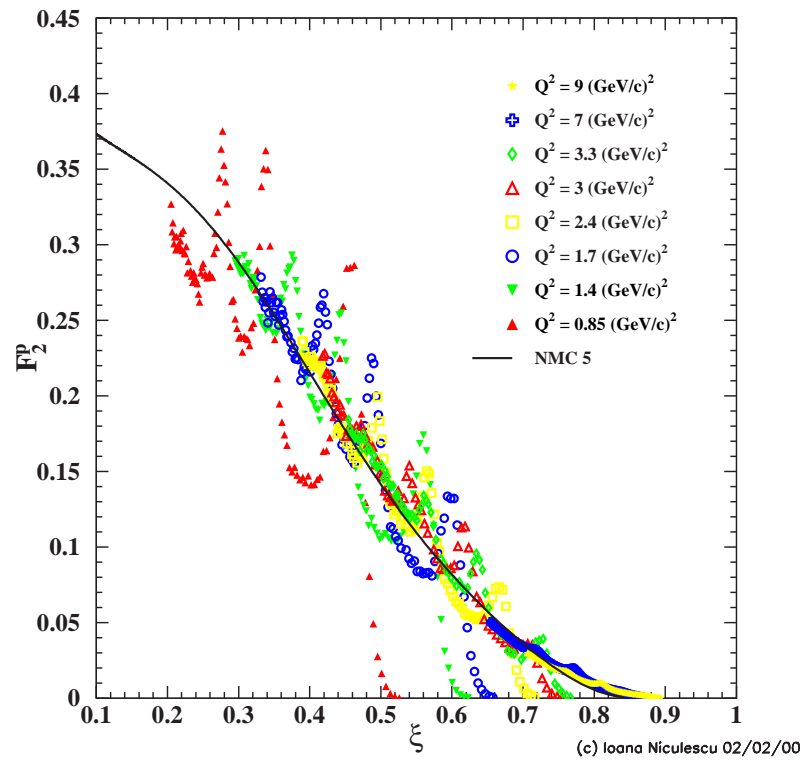

Fig. 1. Recent data for the structure function $F_{2}^{p}$ from Jefferson Lab[2]. structure from the structure function. Clearly the data up to $Q^{2}=9 \mathrm{GeV}^{2}$ continue to show evidence of resonance structure. However, the data appears to oscillate about the scaling curve. This phenomenon is called Bloom-Gilman duality.

Bloom-Gilman duality seems to be puzzling since scaling is often assumed to be associated with asymptotic freedom which allows quarks to be treated as essentially free particles at high energies where the QCD coupling becomes small. Bloom-Gilman duality shows however that the scaling behavior seems to remain in some average sense even at relatively low $Q^{2}$ where the explicitly nonperturbative resonance behavior is still manifest.

However, it has been shown several times that systems that are not asymptotically free also scale[5-7]. We will examine some simple quantum mechanical models in an effort to explore the minimal quantum mechanical requirements for scaling and duality.

\section{Energy-Weighted Sum Rules}

First consider the case of a single nonrelativistic particle in a potential well with the Schödinger hamiltonian

$$
H=\frac{p^{2}}{2 m}+V(x)
$$

The longitudinal response function for this case can be written as

$$
R_{L}(q, \nu)=\int_{-\infty}^{\infty} \frac{d t}{2 \pi} e^{i \nu t}<\psi_{0}\left|e^{i H t} e^{-i \boldsymbol{q} \cdot \boldsymbol{x}} e^{-i H t} e^{i \boldsymbol{q} \cdot \boldsymbol{x}}\right| \psi_{0}>
$$


Applying the momentum shift operator, the response function can then be written as

$$
R_{L}(q, \nu)=\int_{-\infty}^{\infty} \frac{d t}{2 \pi} e^{i \nu t}<\psi_{0}\left|e^{i H t} e^{-i\left(\frac{q^{2}}{2 m}+\frac{q p_{z}}{m}+H\right) t}\right| \psi_{0}>
$$

For us to be able to find a scaling function it is necessary that we be able to find a scaling variable that the area, position and width of the structure function are fixed at large momentum transfers. We can examine the characteristics of this model be considering the Energy-Weighted Sum Rules(EWSR) or energy moments of the response function defined as:

$$
\begin{aligned}
S_{n}(\boldsymbol{q}) & =\int_{-\infty}^{\infty} d \nu \nu^{n} R_{L}(\boldsymbol{q}, \nu) \\
& =\left(i \frac{\partial}{\partial t}\right)^{n}<\psi_{0}\left|e^{i H t} e^{-i\left(\frac{q^{2}}{2 m}+\frac{q p_{z}}{m}+H\right) t}\right| \psi_{0}>_{t=0}
\end{aligned}
$$

For a spherically symmetrical ground state the first three EWSR are then

$$
\begin{aligned}
& S_{0}(q)=1 \\
& S_{1}(q)=\frac{q^{2}}{2 m}=<\nu> \\
& S_{2}(q)=\frac{q^{4}}{4 m^{2}}+\frac{q^{2}}{m^{2}}<\psi_{0}\left|p_{z}^{2}\right| \psi_{0}>=<\nu^{2}>
\end{aligned}
$$

$S_{1}$ and $S_{2}$ can be use to calculate the mean square width as

$$
(\Delta \nu)^{2}=<\nu^{2}>-<\nu>^{2}=\frac{q^{2}}{m^{2}}<\psi_{0}\left|p_{z}^{2}\right| \psi_{0}>
$$

Note that the area is unity as the result of the Coulomb Sum Rule, and the position and width depend only on the interaction through the ground state wave functions. This suggests that it should be possible to obtain a scaling function by the choice of an appropriate scaling variable.

\section{3 y-Scaling}

The nonrelativistic scaling variable $y[8]$ can be obtained by considering a noninteracting gas of particles characterize by a momentum distribution $n(p)$. The longitudinal response function in this case is

$$
\begin{aligned}
R_{L}(q, \nu) & =\int \frac{d^{3} p}{(2 \pi)^{3}} n(p) \delta\left(\nu+\frac{\boldsymbol{p}^{2}}{2 m}-\frac{(\boldsymbol{p}+\boldsymbol{q})^{2}}{2 m}\right) \\
& =\frac{m}{4 \pi^{2} q} \int_{|y|}^{\infty} d p p n(p)=\frac{m}{q} \mathcal{F}(y)
\end{aligned}
$$

where

$$
y=\frac{m}{q} \nu-\frac{q}{2}
$$

is the minimum value of the initial momentum and $\mathcal{F}(y)$ is the scaling function.
Solving (8) for $\nu$, substituting it into (3) and rescaling the time variable to $\tau=\frac{q}{m} t$, we obtain the "scaling function"

$$
\mathcal{F}(q, y)=\int_{-\infty}^{\infty} \frac{d \tau}{2 \pi} e^{i y \tau}<\psi_{0}\left|e^{i \frac{m}{q} H \tau} e^{-i\left(p_{z}+\frac{m}{q} H\right) \tau}\right| \psi_{0}>.
$$

Note that the interaction potential now appears only in combination with a power of $\frac{1}{q}$.

A new set of y-weighted sum rules can be defined as

$$
\begin{aligned}
\mathcal{S}_{n}(q) & \equiv \int_{-\infty}^{\infty} d y y^{n} \mathcal{F}(q, y) \\
& =\left(i \frac{\partial}{\partial \tau}\right)^{n}<\psi_{0}\left|e^{i \frac{m}{q} H \tau} e^{-i\left(p_{z}+\frac{m}{q} H\right) \tau}\right| \psi_{0}>_{\tau=0}
\end{aligned}
$$

The four lowest $\mathrm{y}$-weighted sum rules are:

$$
\begin{aligned}
& \mathcal{S}_{0}(q)=1 \\
& \mathcal{S}_{1}(q)=<\psi_{0}\left|p_{z}\right| \psi_{0}>=0 \\
& \mathcal{S}_{2}(q)=<\psi_{0}\left|p_{z}^{2}\right| \psi_{0}> \\
& \mathcal{S}_{3}(q)=<\psi_{0}\left|\left(\hat{p}_{z}^{3}+\frac{m}{q}\left(\hat{p}_{z} V(r)\right) \hat{p}_{z}\right)\right| \psi_{0}>
\end{aligned}
$$

Note that the first three of these sum rules show that the area, position and width of the scaling function are independent of $q$ and depend upon the potential only through the ground state wave function. The fourth moment contains some interaction dependence. However, this goes as $\frac{1}{q}$ and will therefore vanish for large q. Indeed, this will also be the case for all higher moments. This shows that this kind of model satisfies the conditions for scaling independent of the potential.

Now consider the example of a confining potential where only bound states exist. In this case the longitudinal response is

$$
R_{L}(q, \nu)=\sum_{f}\left|F_{f 0}(q)\right|^{2} \delta\left(\nu+E_{0}-E_{f}\right)
$$

where $F_{f 0}(q)$ is the inelastic form factor for exciting the particle from the ground state to some state $f$. Since all of the states are infinitely narrow, some kind of smoothing must be introduced in order to study the scaling properties of this model. For example,response function can be smoothed by folding with a gaussian of unit area and fixed width $\epsilon$ to give

$$
\bar{R}_{L}(q, \nu)=\frac{1}{\sqrt{\pi} \epsilon} \int_{-\infty}^{\infty} d \nu^{\prime} e^{-\frac{\left(\nu-\nu^{\prime}\right)^{2}}{\epsilon^{2}}} R_{L}\left(q, \nu^{\prime}\right)
$$

The scaling function for the oscillator potential is shown in Figs. 2 and 3 for a variety of momentum transfers up to $q=16 \mathrm{GeV}$ for $\epsilon=0.1 \mathrm{GeV}$ and $0.15 \mathrm{GeV}$ respectively. In each case, the solid line represents the asymptotic case calculated assuming that the final state particle is free. Fig. 


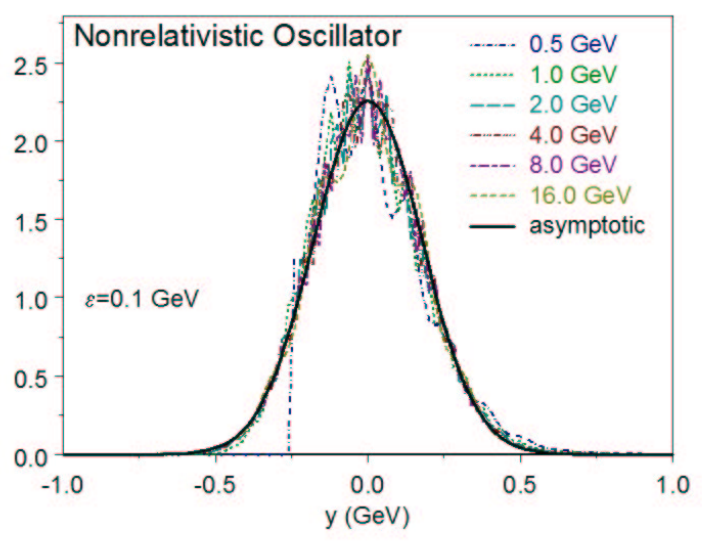

Fig. 2. Scaling function for the nonrelativistic oscillator potential with a folding width of $\epsilon=0.1 \mathrm{GeV}$.

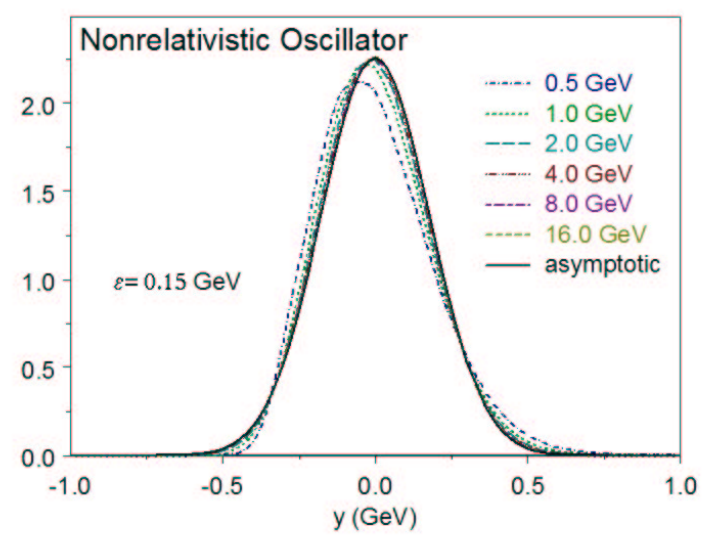

Fig. 3. Scaling function for the nonrelativistic oscillator potential with a folding width of $\epsilon=0.15 \mathrm{GeV}$.

2 clearly shows the qualitative features of Bloom-Gilman duality while the greater width used in Fig. 3 allows the approach to scaling to be examined. This rate of approach to scaling is due to the size of the $\frac{1}{q}$ dependence of the interaction terms.

\section{A Relativistic Model: The Single-Particle Klein-Gordon Equation}

If we are to approach the usual description of Bjorken scaling, it is necessary to examine relativistic models. A simple example of such a model $[9,10]$ is a one-body KleinGordon equation of the form

$$
\left(\boldsymbol{p}^{2}+m^{2}+V^{2}(\boldsymbol{x})\right)\left|\psi>=E^{2}\right| \psi>.
$$

This model is particularly attractive since the solution to this equation can be found by rewriting the equation to resemble a Schödinger equation as

$$
\left(\frac{\boldsymbol{p}^{2}}{2 m}+\frac{V^{2}(\boldsymbol{x})}{2 m}\right)\left|\psi>=\frac{E^{2}-m^{2}}{2 m}\right| \psi>.
$$

If we now identify a "nonrelativistic" potential as

$$
V_{N R}(\boldsymbol{x})=\frac{V^{2}(\boldsymbol{x})}{2 m}
$$

and a "nonrelativistic" energy as

$$
E_{N R}=\frac{E^{2}-m^{2}}{2 m},
$$

the relativistic wave functions are the same as the nonrelativistic wave functions provided that care is taken to include the necessary energy factors to properly normalize the Klein-Gordon wave functions, and the relativistic energy is given by

$$
E= \pm \sqrt{2 m E_{N R}+m^{2}} .
$$

It is possible to proceed to construct EWSR in exactly the same manner as in the nonrelativistic case. The only technical complication is the necessity of defining a hamiltonian for the Klein-Gordon equation which can be accomplished by using a two-component formalism[11]. However, the interpretation of these sum rules in geometrical terms is not as straightforward as in the nonrelativistic case due to the presence of the negative energy solutions to the one-body Klein-Gordon equation.

It is easiest to see how the situation is complicated by considering the case where the potential is chosen such that only bound states occur. The longitudinal response for this case is

$$
\begin{aligned}
R_{L}(q, \nu)= & \sum_{f} \frac{\left(\nu+2 E_{0}\right)^{2}}{4 E_{0} E_{f}}\left|F_{f 0}(q)\right|^{2} \\
& \times\left(\delta\left(\nu+E_{0}-E_{f}\right)-\delta\left(\nu+E_{0}+E_{f}\right)\right)(19)
\end{aligned}
$$

where the presence of positive and negative energy contributions is explicit.

Figures 4, 5 and 6 show the numerical calculation of the first three EWSR of this response for the case where the potential is a linear confining potential. This results in wave functions that are identical to the nonrelativistic oscillator wave functions. In these figures the positive and negative energy contributions are displayed separately. Figure 4 represents $S_{0}$. The total contribution of both positive and negative contributions (dashed line) has the constant value of 1 , as would be expected since this sum rule is the Coulomb sum rule and should be equal to the square of the charge. Note however that this is made up of a positive energy contribution (solid line) and a negative energy contribution (dash-dotted line) of opposite signs. Both contributions increase in magnitude with $q$. For comparison the positive energy contribution of the PWIA (dashed-triple-dotted line) is included and can be seen to approach the positive energy oscillator result with increasing $q$. 


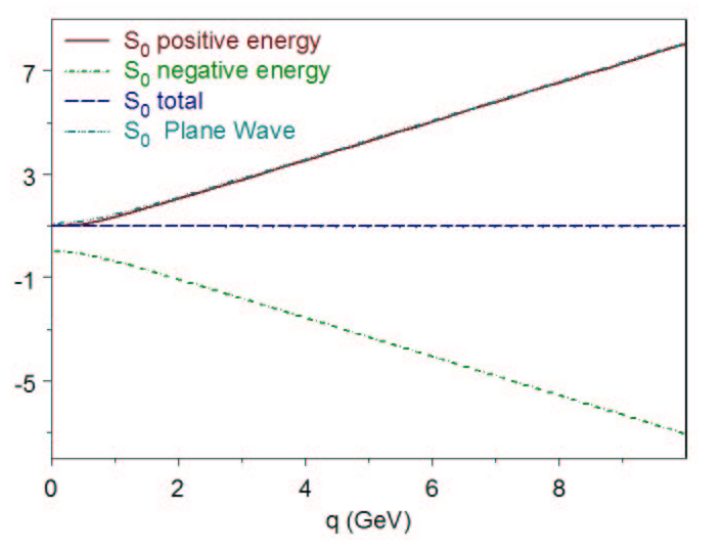

Fig. 4. Positive and negative energy contributions to the Coulomb sum rule for the relativistic oscillator model.

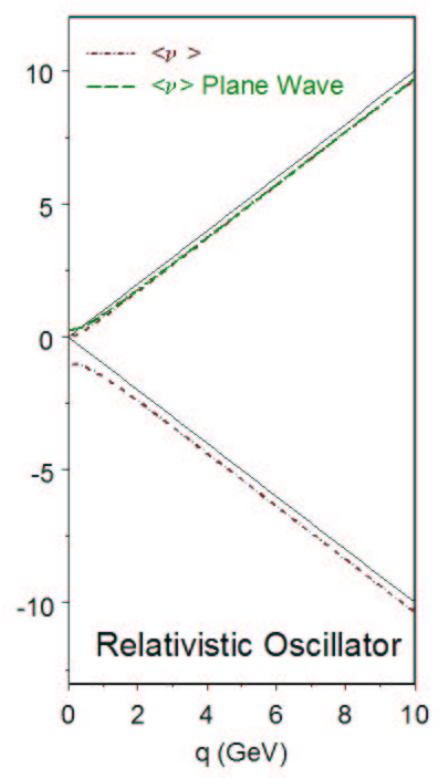

Fig. 5. Positive and negative energy contributions to the average energy transfer for the relativistic oscillator model.

Figure 5 shows the contribution to $S_{1}=\langle\nu\rangle$. Here the solid lines represent $Q^{2}=0$ and the dash-dotted lines are the positive and negative energy contributions to the average value of $\nu$. The positive energy contribution is centered in the spacelike region while the negative energy contribution is centered in the negative-energy timelike region. In both cases, $\langle\nu\rangle$ becomes linear at larger values of $q$ and becomes parallel to the light cone. The positive energy contribution for the PWIA is also shown and except for small values of $q$ is close to the oscillator result.

Figure 6 shows the mean square widths of the positive (solid line) and negative energy (dash-dotted line) con-

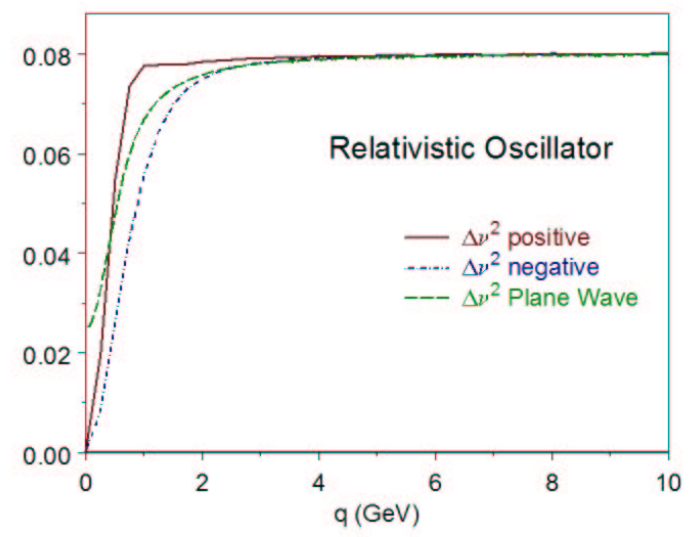

Fig. 6. Positive and negative energy contributions to the mean-square width of the response in the relativistic oscillator model.

tributions. In both cases the width approaches the same constant value as does the positive energy PWIA (dashed line).

These figures indicate that the positions of the positive and negative energy peaks move in opposite directions as $q$ increases while the widths of the peaks approach the same constant value. The areas under these peaks increase in magnitude linearly for large $q$ with the positive energy contribution being positive and the negative being negative. The y-scaling variable can be chosen to either fix the position of the positive or negative energy peaks, but not both. Since the negative energy contribution is clearly not physical, we will choose this variable to fix the position of the positive energy peak. The scaling function must also be chosen such that the area of the positive energy peak to the structure function is fixed.

A y-scaling variable can be obtained by considering the response of a relativistic gas of particles in analogy to the nonrelativistic case. This leads to the variable[12]

$$
y=\nu \sqrt{1+\frac{4 m^{2}}{q^{2}-\nu^{2}}}-q .
$$

Note that this maps the interval $-\infty<y<\infty$ onto the spacelike region. It should be mentioned that if a system displays y-scaling at fixed $q$ then it will also display xscaling at fixed $Q^{2}$. Indeed there is a direct relationship between $\mathrm{y}$ and $\mathrm{x}$ variables and the $\mathrm{y}$ variable used here corresponds to the $\mathrm{x}$ variable derived by Barbieri, et al.[13]

The scaling function appropriate to the Klein-Gordon equation is the longitudinal contribution to $q W_{2}[10]$ which gives

$$
\mathcal{F}(q, y)=q \frac{\left(q^{2}-\nu^{2}\right)^{2}}{q^{4}} R_{L}(q, \nu(q, y)) .
$$

Figure 7 shows the scaling function for the relativistic oscillator for a variety of momentum transfers. The asymptotic result is shown as a solid line. This clearly shows the qualitative features of Bloom-Gilman duality, 


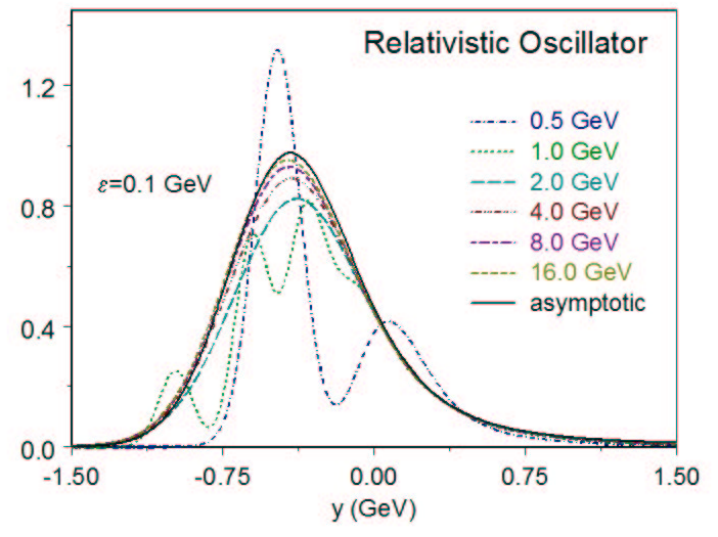

Fig. 7. Scaling function for the relativistic oscillator model for a range of momentum transfers.

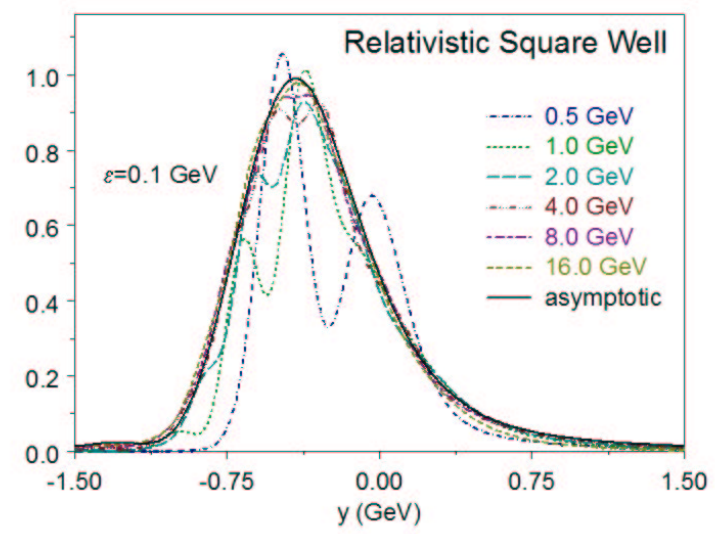

Fig. 8. Scaling function for the relativistic square well model for a range of momentum transfers.

however the approach to scaling is slower than in the nonrelativistic case. Note that the scaling function is peaked at approximately $y=-0.5 \mathrm{GeV}$ rather than at $y=0$ as in the nonrelativistic case. This is largely the result of the $q$ and $\nu$ dependent factors in the definition of the scaling function.

Figure 8 shows the scaling function for a relativistic infinite spherical well potential. Again the qualitative features of duality appear although the rate of approach to scaling is somewhat faster. This suggests that scaling is a feature of this model but the rate of scaling depends upon the dynamics associated with the choice of potential.

\section{Summary}

We have examined two simple models, one nonrelativistic and one relativistic, that scale and have the qualitative features of Bloom-Gilman duality. These models contain only bound states and are not asymptotically free in the sense of perturbative QCD. This suggests that scaling and duality are more general features of quantum mechanics and are not necessarily associated with QCD. However the dynamics of QCD should affect the rate at which scaling and duality occur. By showing that duality and scaling are not necessarily associate with QCD, these models make Bloom-Gilman duality appear considerably less miraculous.

We are currently extending these calculations to a oneparticle Dirac equation and are exploring the general conditions under which scaling should occur in potential models.

This work was supported in part by funds provided by the U.S. Department of Energy (D.O.E.) under cooperative research agreement No. DE-AC05-84ER40150 and by the National Science Foundation under grant No. PHY-0139973.

\section{References}

1. E. D. Bloom and F. J. Gilman, Phys. Rev. Lett. 25 (1970) 1140; E. D. Bloom and F. J. Gilman, Phys. Rev. D4 (1971) 2901.

2. I. Niculescu et al., Phys. Rev. Lett. 85, 1182 (2000); 85 (2000) 1186; R. Ent, C.E. Keppel and I. Niculescu, Phys. Rev. D62 (2000) 073008; Phys. Rev. D64 (2001) 038302.

3. O. Nachtmann, Nucl. Phys. B63 (1973) 237; O. Nachtmann, Nucl. Phys. B78 (1974) 455.

4. O. W. Greenberg and D. Bhaumik, Phys. Rev. D4 (1971) 2048.

5. O. W. Greenberg, Phys. Rev. D47 (1993) 331.

6. S. A. Gurvitz and A. S. Rinat, Phys. Rev. C47 (1993) 2901; S. A. Gurvitz, Phys. Rev. C52 (1995) 1433.

7. E. Pace, G. Salme, and F. M. Lev, Phys. Rev. C57 (1998) 2655.

8. G. B. West, Phys. Rep. 18 (1975) 263.

9. N. Isgur, S. Jeschonnek, W. Melnitchouk, and J. W. Van Orden, Phys. Rev. D64 (2001) 054005.

10. S. Jeschonnek, J. W. Van Orden, Phys. Rev. D65 (2002) 094038.

11. Franz Gross, Relativistic Quantum Mechanics and Field Theory (Wiley-Interscience, New York 1993) 102.

12. W. M. Alberico, A. Molinari, T. W. Donnelly, E. L. Kronenberg, and J. W. Van Orden, Phys. Rev. C38 (1988) 1801.

13. R. Barbieri, J. Ellis, M. K. Gaillard, and G. G. Ross, Phys. Lett. 64B (1976) 171; R. Barbieri, J. Ellis, M. K. Gaillard, and G. G. Ross, Nucl. Phys. B117 (1976) 50. 